\title{
Online Balancing of Range-Partitioned Data with Applications to Peer-to-Peer Systems
}

\author{
Prasanna Ganesan \\ Mayank Bawa \\ Hector Garcia-Molina \\ Stanford University \\ Stanford, CA 94305 \\ \{prasannag, bawa,hector\}@cs.stanford.edu
}

\begin{abstract}
We consider the problem of horizontally partitioning a dynamic relation across a large number of disks/nodes by the use of range partitioning. Such partitioning is often desirable in large-scale parallel databases, as well as in peer-to-peer (P2P) systems. As tuples are inserted and deleted, the partitions may need to be adjusted, and data moved, in order to achieve storage balance across the participant disks/nodes. We propose ef£cient, asymptotically optimal algorithms that ensure storage balance at all times, even against an adversarial insertion and deletion of tuples. We combine the above algorithms with distributed routing structures to architect a P2P system that supports effcient range queries, while simultaneously guaranteeing storage balance.
\end{abstract}

\section{Introduction}

The problem of partitioning a relation across multiple disks has been studied for a number of years in the context of parallel databases. Many shared-nothing parallel database systems use range partitioning to decluster a relation across the available disks for performance gains [8, 10,28]. For example, transactions in OLTP systems often access tuples associatively, i.e., all tuples with a specifc attribute value, or a small range of values. Range partitioning ensures that a transaction requires data only from a single disk (most of the time), thus enabling inter-query parallelism and nearlinear speed-up [11].

A well-known concern in range partitioning is skew, where only a few partitions (disks/nodes) are involved in

Permission to copy without fee all or part of this material is granted provided that the copies are not made or distributed for direct commercial advantage, the VLDB copyright notice and the title of the publication and its date appear, and notice is given that copying is by permission of the Very Large Data Base Endowment. To copy otherwise, or to republish, requires a fee and/or special permission from the Endowment.

Proceedings of the 30th VLDB Conference,

Toronto, Canada, 2004 the execution of most queries. Skew can be classifed into (a) data skew, where data may be unequally distributed across the partitions, and (b) execution skew, where data accesses may not be uniform across the partitions [11]. As the relation evolves over time, or as workloads change, both data and execution skew pose a serious problem.

Today's database systems put the onus on administrators to monitor performance and re-partition data whenever the skew becomes "too large", an approach fraught with diffculties. In contrast, we consider online load-balancing solutions, which dynamically move data across nodes and avoid skew at all times. Online load-balancing promises three major advantages over periodic manual re-partitions: (a) a consistently effcient $24 / 7$ operation by eliminating performance degradation between, and system hiccups during, manual re-partitions; (b) a simplifed control panel by eliminating partition confguration from the administrator's list of chores; and (c) a smaller cost especially in systems with a high degree of parallelism, where even a few inserts/deletes may cause a large skew.

Skew can be characterized by the imbalance ratio $\sigma$ defned as the ratio of the loads of the largest and smallest partitions in the system. In order to ensure that $\sigma$ is small, data may have to be moved from one disk/node to another as the relation grows or shrinks. Thus a key requirement for a load balancing algorithm is to minimize the number of tuples moved in order to achieve a desired $\sigma$.

Summary of Results In this paper, we focus on algorithms for eliminating data skew to achieve storage balance, although our algorithms can be generalized to handle execution skew as well. Our load-balancing algorithms guarantee that $\sigma$ is always bounded by a small constant $c$. The bound $c$ is, in fact, a tunable parameter that can be set to values as low as 4.24. Moreover, each insert or delete of a tuple is guaranteed to require just an (amortized) constant number of tuple movements, even against an adversarial sequence of inserts and deletes. Thus, our algorithms offer storage balance at all times, against all data distributions, while ensuring that the overhead is asymptotically optimal, and often much less than that of periodic repartitioning. 
Application to P2P Systems Our online load balancing algorithms are motivated by a new application domain for range partitioning: peer-to-peer (P2P) systems. P2P systems store a relation over a large and dynamic set of nodes, and support queries over this relation. Many current systems, known as Distributed Hash Tables (DHTs) [22, 23, 27], use hash partitioning to ensure storage balance, and support point queries over the relation.

There has been considerable recent interest in developing P2P systems that can support effcient range queries [3, $4,25]$. For example, a P2P multi-player game might query for all objects located in an area in a virtual 2-D space. In a P2P web cache, a node may request (pre-fetch) all pages with a specifc URL pre£x. It is well-known [5] that hash partitioning (and hence a DHT) is ineffcient for answering such ad hoc range queries, motivating a search for new networks that allow range partitioning while still maintaining the storage balance offered by normal DHTs.

The P2P domain throws up its own challenges for rangepartitioning and load balancing. Nodes in a P2P system may arrive and depart at will; we therefore require load balance over such a dynamic set of nodes. In addition, $\mathrm{P} 2 \mathrm{P}$ systems are decentralized, necessitating the design of distributed data structures for maintaining partition information. We show how to enhance our online loadbalancing algorithm with overlay-network structures to architect a new P2P system whose performance is asymptotically identical to that of DHTs, but with the advantage of enabling effcient range queries.

Organization We defne the online load-balancing problem for parallel databases in Section 2. We present our load-balancing algorithm and analyze it in Section 3. We adapt our algorithm to a P2P setting in Section 4. We experimentally evaluate our algorithms in Section 5. We discuss related work in Section 6.

\section{Problem Setup and Basic Operations}

We will now defne a simple abstraction of a parallel database, discuss a cost model for load balancing in this context, and defne two basic operations used by loadbalancing algorithms. We defer a discussion of P2P systems to Section 4.

\subsection{Setup}

We consider a relation divided into $n$ range partitions on the basis of a key attribute, with partition boundaries at $R_{0} \leq$ $R_{1} \leq \ldots \leq R_{n}$. Node $N_{i}$ manages the range $\left[R_{i-1}, R_{i}\right)$, for all $0<i \leq n$. When $R_{i-1}=R_{i}, N_{i}$ is said to manage the empty partition $\left[R_{i-1}, R_{i}\right)$. Nodes managing adjacent ranges are said to be neighbors. We let $L\left(N_{i}\right)$ denote the load at $N_{i}$, de£ned to be the number of tuples stored by $N_{i}$. We assume a central site has access to the range-partition information $\left[R_{0}, R_{1}, \ldots, R_{n}\right]$ and directs each query, insert and delete to the appropriate node(s).

Each insert or delete of a tuple is followed by an execution of the load-balancing algorithm which may pos- sibly move data across nodes. The load-balancing algorithms we consider are local in that the algorithm executes only on the node at which the insert or delete occurs. For now, we ignore concurrency control issues (see Section 3.4), and consider only the equivalent serial schedule of inserts and deletes, interleaved with the executions of the load-balancing algorithm.

Imbalance Ratio A load-balancing algorithm guarantees an imbalance ratio $\sigma$ if, after the completion of every insert or delete operation and its corresponding load-balancing step, $\max _{i} L\left(N_{i}\right) \leq \sigma \min _{i} L\left(N_{i}\right)+c_{0}$, for some £xed constant $c_{0}$. As is conventional, we have defned $\sigma$ as the asymptotic ratio between the largest and smallest loads.

\subsection{Costs of Load Balancing}

Data Movement All load-balancing algorithms will need to move data from one node to another in order to achieve balance. We use a simple linear cost model, where moving one tuple from any node to any other node costs one unit. Such a model reasonably captures both the networkcommunication cost of transferring data, as well as the cost of modifying local data structures at the nodes.

Partition Change Data movement is accompanied by a change in the partition boundaries. The central site needs to be informed to enable it to correct its partition information $\left[R_{0}, R_{1}, \ldots, R_{n}\right]$. Notice that the movement of a tuple may cause a change in at most one partition boundary, resulting in at most one update message to the central site. We can thus absorb this cost into the data movement cost itself.

Load Information Finally, the load-balancing algorithm that executes locally at a node may require non-local information about the load at other nodes. For now, we assume that the central site keeps track of the load at each node, thus requiring each node to inform the site after a successful insert, delete or data movement. A node that needs load information can simply contact the central site at any time to obtain it. We can thus absorb this cost into the cost of tuple insert, delete and movement as well.

In summary, we measure the cost of a load-balancing algorithm simply as the number of tuples moved by the algorithm per insert or delete. Our interest is in the amortized cost per insert or delete, for adversarial (worst-case) sequences of insertions and deletions. The amortized cost of an insert or delete is said to be $c$ if, for any sequence of $t$ tuple inserts and deletes, the total number of tuples moved is at most $t c$.

Problem Statement Develop a load balancing algorithm which guarantees a constant imbalance ratio $\sigma$ with low amortized cost per tuple insert and delete.

We will show that it is possible to achieve a constant $\sigma$ while ensuring that the amortized cost per insert and delete is also a constant. Such an algorithm is asymptotically optimal since, for any load-balancing algorithm, there exist sequences of $t$ operations that require $\Omega(t)$ tuple movements to ensure load balance. 


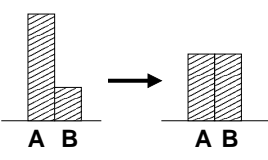

(a)

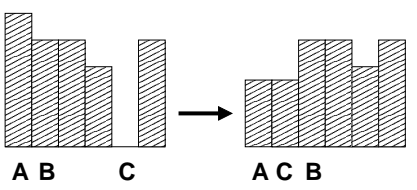

(b)
Figure 1: (a) NBRAdJUST involving $A$ and $B$ and (b) REORDER involving $A$ and $C$. The height of a bar represents the load of the corresponding node.

\section{3 “Universal” Load-Balancing Operations}

What operations can be used to perform load balancing? An intuitive operation is as follows: when a node becomes responsible for too much data, it can move a portion of its data to its neighbor and thus attempt to balance out the load. We call such an operation NBRADJUST which is illustrated in Figure 1(a) and de£ned below.

NBRAdJust $A$ pair of neighboring nodes $N_{i}$ and $N_{i+1}$ may alter the boundary $R_{i}$ between their ranges by transferring data from one node to the other. ${ }^{1}$

A load-balancing algorithm can be devised based on just this operation, e.g. [12, 18]. However, such an algorithm is provably expensive as we show in the following theorem. ${ }^{2}$

Theorem 1. Any load-balancing algorithm, deterministic or randomized, that uses only NBRADJUST and guarantees a constant imbalance ratio $\sigma$, has amortized cost $\Omega(n)$ per insert and delete.

The above theorem shows that any algorithm that uses only NBRADJUST would incur a cost per insert that is at least linear in the number of nodes. In contrast, our goal is to achieve a constant cost per insert. The key to ef£cient load balancing lies in a second operation, REORDER, illustrated in Figure 1(b) and de£ned below.

REORDER $A$ node $N_{i}$ with an empty range $\left[R_{i}, R_{i}\right)$ changes its position and splits the range $\left[R_{j}, R_{j+1}\right)$ managed by a node $N_{j}: N_{j}$ now manages range $\left[R_{j}, X\right)$ while $N_{i}$ takes over $\left[X, R_{j+1}\right)$ for some value of $X, R_{j} \leq X \leq$ $R_{j+1}$. The nodes are re-labeled appropriately.

EXAMPLE 2.1. Consider the scenario shown in Figure 2, where node A has 100 tuples, the next three nodes $(B, C, D)$ have 60 tuples each, while the last two $(E, F)$ have 20 tuples each. The least expensive scheme to improve load balance while preserving key ordering is to transfer all 20 tuples from $E$ to $F$, and then use REORDER to split the load of $A$ between $A$ and $E$. The cost of such a scheme is 70 tuple movements; in contrast, a NBRADJUST-based balancing requires 250 tuple movements.

It turns out that the REORDER operation is not only necessary, but also suf£cient for ef£cient load balancing. In

\footnotetext{
${ }^{1}$ In the extreme case when $N_{i}$ takes over the entire range, $\left[R_{i-1}, R_{i+1}\right), N_{i+1}$ is assigned the empty range $\left[R_{i+1}, R_{i+1}\right)$.

${ }^{2}$ Proofs omitted in this paper are available in a technical report [13].
}

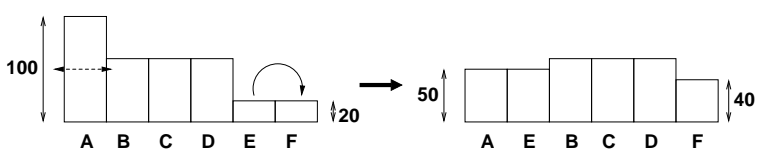

Figure 2: The cost of load balancing using REORDER is 70 while using successive NBRADJUST operations costs 250.

fact, we show below that the operations NBRADJUST and REORDER are universal in that they can together be used to ef£ciently implement any load-balancing algorithm.

Theorem 2. Given a load-balancing algorithm $A$, it is possible to construct a new algorithm $\widehat{A}$ that uses only the NBRADJUST and REORDER operations such that, for any sequence of $t$ inserts and deletes,

(a) Both $A$ and $\widehat{A}$ achieve identical load distribution.

(b) The cost of $\widehat{A}$ is at most the cost of $A$.

\section{Algorithms for Load Balancing}

Consider the following approach for load balancing: a node attempts to shed its load whenever its load increases by a factor $\delta$, and attempts to gain load when it drops by the same factor. Formally, we consider an infnite, increasing geometric sequence of thresholds $T_{i}=\left\lfloor c \delta^{i}\right\rfloor$, for all $i \geq 1$ and some constant $c$. When a node's load crosses a threshold $T_{j}$, the node initiates a load-balancing procedure. We call such an approach the Threshold Algorithm.

\subsection{The Doubling Algorithm}

We start with the special case $\delta=2$, and the thresholds $T_{i}=2^{i-1}$. We begin by considering tuple insertions. Every time a node's load ${ }^{3}$ increases to a value $T_{i}+1$, the node initiates ADJUSTLOAD speci£ed in Procedure 1.

The load-balancing procedure ADJUSTLOAD is quite simple. When node $N_{i}$ 's load increases beyond a threshold, it £rst (lines 3-6) attempts to perform NBRADJUST with its lightly-loaded neighbor, say $N_{i+1}$, by averaging out its load with $N_{i+1}$. If both neighbors have high load (more than half that of $N_{i}$ ), $N_{i}$ attempts to perform REORDER with the globally least-loaded node $N_{k}$ (lines 812). If $N_{k}$ 's load is small enough (less than a quarter of $N_{i}$ ), $N_{k}$ sheds all its data to $N_{k \pm 1}$, and takes over half the load of $N_{i}$. If $N_{i}$ is unable to perform either NBRADJUST or REORDER, $N_{i}$ concludes that the system load is indeed balanced and performs no data movement.

Note that when $N_{i}$ initiates either a NBRADJUST or REORDER, there is a corresponding recursive invocation of AdJUSTLOAD at node $N_{i+1}$ or $N_{k \pm 1}$ respectively. Frequently, these recursive invocations do not necessitate any further data movement; even if data movement is necessary, we can show that such data movement would utilize only NBRADJUST. Similarly, there is also a recursive invocation

\footnotetext{
${ }^{3}$ For technical reasons, we de£ne $L^{\prime}(N)=T_{1}+L(N)$, and use $L^{\prime}$ as the node load. Note that the same guarantees on $\sigma$ hold when using either $L$ or $L^{\prime}$; for notational convenience, we let $L$ denote this new defnition of load.
} 
of ADJUSTLOAD at node $N_{i}$ itself (line 6); this invocation is necessary only in one special case - when ADJUSTLOAD is being executed at $N_{k \pm 1}$ after a REORDER - and is also guaranteed to utilize only NBRADJUST.

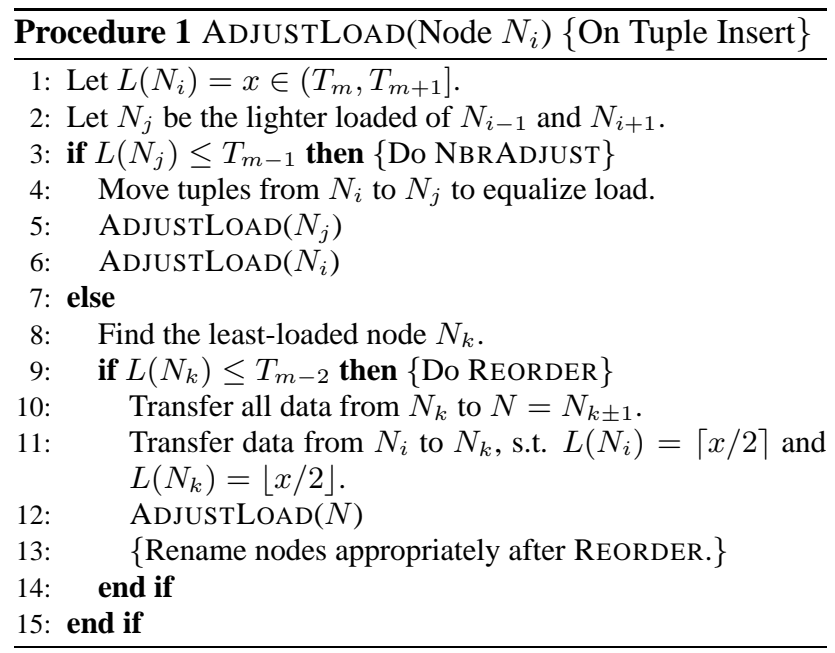

Deletions are handled in a symmetric fashion. When a node's load drops to a threshold $T_{j}=2^{j}$, it frst attempts NBRADJUST with a neighbor, if the neighbor's load is larger than $T_{j+1}=2^{j+1}$. Otherwise, it attempts to REORDER itself and split the highest-loaded node $N_{k}$ in the system, if $N_{k}$ 's load is more than $T_{j+2}$.

We will show later that the Doubling Algorithm ensures that $\sigma=8$, while the amortized cost of tuple insert and and delete is constant. However, it is possible to reduce $\sigma$ further by generalizing this Doubling Algorithm.

\subsection{The General Threshold Algorithm}

The Doubling Algorithm set $\delta=2$ and triggered load balancing when a node's load changed by a factor 2 to obtain $\sigma=8$. A natural question, then, is to ask whether the algorithm generalizes to other values of $\delta$, and whether it is possible to obtain a better $\sigma$ by using a smaller $\delta$ value.

The Doubling algorithm generalizes to allow $\delta$ to be any real number greater than or equal to the golden ratio $\phi=(\sqrt{5}+1) / 2 \simeq 1.62$. For any real number $\delta \geq \phi$, we may de£ne a general Threshold Algorithm as follows: We defne a threshold sequence of $T_{i}=\left\lfloor c \delta^{i}\right\rfloor$, for an appropriately chosen constant $c>0$. Each node is required to execute Procedure ADJUSTLOAD, every time its load crosses a threshold. This Threshold Algorithm guarantees $\sigma=\delta^{3}$ with a constant cost per tuple insert and delete.

The Fibbing Algorithm: An extreme of the general Threshold Algorithm arises when $\delta=\phi$, for which we may de£ne a variant called the Fibbing Algorithm. This algorithm de£nes the set of thresholds $T_{i}$ to be the Fibonacci numbers (with $T_{1}=1$ and $T_{2}=2$ ). As we prove in Section 3.3, the Fibbing Algorithm guarantees an imbalance ratio of $\phi^{3} \simeq 4.24$.

\subsection{Analysis}

We now present an analysis of the Threshold algorithm (and the Fibbing algorithm), both in terms of the guaranteed imbalance ratio, and in terms of the cost of insert and delete. Our analysis relies on seven properties of the threshold sequence that is satis£ed both by Fibonacci numbers, and by threshold sequences of the form $T_{i}=\left\lfloor c \delta^{i}\right\rfloor$, allowing the same analysis to apply to both the Threshold algorithm and the Fibbing algorithm. We summarize these properties in the following lemma.

Lemma 1. If $T_{i}=\left\lfloor c \delta^{i}\right\rfloor$, for a suitably large $c$ and $\delta \geq$ $\phi$, the following properties hold for all $r \geq 1$. The same properties hold if $T_{i}$ is the $i^{\text {th }}$ Fibonacci number $\left(T_{i}=\right.$ $\left[c \delta^{i}\right]$, with $c=\phi / \sqrt{5}$ and $\left.\delta=\phi\right)$.

(a) $\left\lfloor\left(T_{r}+T_{r+2}\right) / 2\right\rfloor \geq T_{r+1}$

(b) $\left\lceil\left(T_{r}+T_{r+1}+1\right) / 2\right\rceil \leq T_{r+1}$

(c) $T_{r}+T_{r+1} \leq T_{r+2}$

(d) $\left\lceil\left(T_{r}+1\right) / 2\right\rceil \leq T_{r}$

(e) $\left\lfloor\left(T_{r+2}+1\right) / 2\right\rfloor>T_{r}$

(f) $T_{r+k}+1 \geq \delta^{k} T_{r} \geq T_{r+k}-C$, where $C=1$ if $T_{i}$ is the $i^{\text {th }}$ Fibonacci number, and $C=\delta^{k}$ otherwise, for all integers $k>0$.

(g) $\left\lfloor\left(T_{1}+T_{2}+1\right) / 2\right\rfloor>T_{1}$

De£nition 3.1. For any node $N$, de£ne $I(N)=r$ if and only if $L(N) \in\left(T_{r-1}, T_{r}\right]$, i.e., $N$ 's load is in the $r^{\text {th }}$ geometric interval.

Theorem 3. The following invariants hold after any sequence of inserts and deletes for the Threshold (and Fibbing) algorithm:

(a) NBRBALANCE: For any pair of neighbors $N_{i}$ and $N_{i+1}, I\left(N_{i}\right) \leq I\left(N_{i+1}\right)+1$.

(b) GloBAlBALANCE: For any pair of nodes $N_{i}$ and $N_{j}$, $I\left(N_{i}\right) \leq I\left(N_{j}\right)+2$.

Before proving the above theorem, we frst establish some lemmas on the properties of the NBRADJUST and REORDER, as well as the execution of ADJUSTLOAD.

Lemma 2. If $I\left(N_{i}\right)=r+2$ and $I\left(N_{i+1}\right)=r$, then NBRAdJUST between $N_{i}$ and $N_{i+1}$ ensures that $I\left(N_{i}\right)=$ $I\left(N_{i+1}\right) \geq r+1$.

Lemma 3. Consider a state of the system where both NBRBALANCE and GLOBALBALANCE invariants hold. If a tuple insert now causes a violation of NBRBALANCE, the consequent execution of ADJUSTLOAD will ensure both NBRBALANCE and GLOBALBALANCE.

Proof. Consider a tuple insert at node $N_{i}$. By de£nition, no NBRBALANCE violation arises unless $L\left(N_{i}\right)$ crosses a threshold. Say $L\left(N_{i}\right)$ crosses threshold $T_{x}$. There may then be a violation of NBRBALANCE between $N_{i}$ and either or both of its neighbors. (There may also be a GLOBALBALANCE violation involving $N_{i}$.) In this case, $N_{i}$ executes a NBRAduUst by Procedure AdJUSTLOAD.

W.l.o.g., say $N_{i}$ performs NBRAdJUST with $N_{i+1}$. First, observe that there are no GLOBALBALANCE violations after this NBRADJUST, by Lemma 1(b). After 
this NBRAdjUst, AdJUSTLOAD is recursively invoked on $N_{i+1}$, which may cause $N_{i+1}$ to perform NBRADJUST with $N_{i+2}$, and trigger $N_{i+2}$ into executing ADJUSTLOAD. This process continues until we reach a node $N_{i+k}$ that does not perform a NBRADJUST, or we reach $N_{n}$.

We show that this sequence of NBRADJUST operations ensures that all NBRBALANCE conditions are satis£ed. (Finally, there are recursive calls to ADJUSTLOAD in line 6 , which do not perform any data movement since there are no violations of NBRBALANCE or GLOBALBALANCE.)

Let $L_{j}(N)$ represent the load at node $N$ after the $j^{\text {th }}$ NBRADJUST operation, and $I_{j}(N)=I(N)$ after the $j^{\text {th }}$ NBRADJUST operation. ( $L_{0}$ is the load before any NBRAdJUST operations take place.) The $j^{t h}$ NBRADJUST operation occurs between nodes $N_{i+j-1}$ and $N_{i+j}$. Thus, the load of $N_{i+j-1}$ remains unchanged after the $j^{t h}$ operation.

We will show by induction that, after $j>0$ NBRADJUST operations,

1. $I_{j}\left(N_{i+k}\right) \leq x$ for all $0 \leq k \leq j$.

2. The only NBRBALANCE violation may be between $N_{i+j}$ and $N_{i+j+1}$.

Base Case: We show the above properties for $j=1$. Initially, the only NBRBALANCE violations may be at $\left(N_{i-1}, N_{i}\right)$ and/or $\left(N_{i}, N_{i+1}\right)$. Recall that $I_{0}\left(N_{i}\right)=x+1$ and, since there is a NBRBALANCE violation, $I_{0}\left(N_{i+1}\right)=$ $x-1$. From the GlobalBalance invariant, we deduce $I_{0}\left(N_{i-1}\right) \leq x+1$.

After the frst NBRADJUST operation, we know by Lemma 2 that $I_{1}\left(N_{i}\right)=I_{1}\left(N_{i+1}\right) \geq x$. Also, $L_{1}\left(N_{i-1}\right)=$ $L_{0}\left(N_{i-1}\right)$, thus showing that neither pair $\left(N_{i-1}, N_{i}\right)$ nor $\left(N_{i}, N_{i+1}\right)$ constitute a NBRBALANCE violation. Since only the loads of $N_{i}$ and $N_{i+1}$ were affected by this operation, the only possible NBRBALANCE violation is between $N_{i+1}$ and $N_{i+2}$. It is also clear that $I_{1}\left(N_{i}\right)=I_{1}\left(N_{i+1}\right) \leq$ $x$ (by Lemma $1(\mathrm{~d})$ ).

Induction Step: Assume, by induction, that after $j$ NBRAdJUST operations, $I_{j}\left(N_{i+j}\right) \leq x$, and the only possible NBRBALANCE violation is at $\left(N_{i+j}, N_{i+j+1}\right)$. If there is no such violation, we are done and ADJUSTLOAD terminates. If there is a violation, a NBRADJUST takes place between $N_{i+j}$ and $N_{i+j+1}$.

GLOBALBALANCE assures us that $I_{0}\left(N_{i+j+1}\right)=$ $I_{j}\left(N_{i+j+1}\right) \geq x-2$. Since there is a NBRBALANCE violation, we may deduce $I_{j}\left(N_{i+j+1}\right)=x-2$ and $L_{j}\left(N_{i+j}\right)=$ $x$. Invoking Lemma 2 , we deduce that $I_{j+1}\left(N_{i+j}\right)=$ $I_{j+1}\left(N_{i+j+1}\right) \geq x-1$.

Since $I_{j}\left(N_{i+j-1}\right) \leq x$ by the induction assumption, there is no violation between $N_{i+j-1}$ and $N_{i+j}$. There is obviously no violation between $N_{i+j}$ and $N_{i+j+1}$, since both loads are in the same interval. The only possible violation might be between $N_{i+j+1}$ and $N_{i+j+2}$, which is permitted under the induction assumption. It is also clear that both $I_{j+1}\left(N_{i+j}\right)$ and $I_{j+1}\left(N_{i+j+1}\right)$ are at most $x$, thus completing the induction step.

The above inductive proof, combined with the fact that the procedure terminates when the last node is reached, shows that there are no NBRBALANCE violations when ADJUSTLOAD terminates.

Lemma 4. Consider a state of the system where both NBRBALANCE and GLOBALBALANCE invariants hold. If a tuple insert now causes a violation of GLOBALBALANCE, the consequent execution of ADJUSTLOAD will ensure both GLOBALBALANCE and NBRBALANCE.

Proof of Theorem 3. We prove that the invariants hold by induction on the length $l$ of the insert/delete sequence. The invariants clearly hold when $l=0$ since all nodes contain one sentinel value.

Assume that the invariants hold for $l=r$. Let the $(r+$ $1)^{s t}$ operation be an insert. If this insert does not violate any invariants, we are done. If not, either NBRBALANCE or GLOBALBALANCE is violated. We have shown that all such violations are £xed by ADJUSTLOAD in Lemmas 3 and 4 respectively.

If the $(r+1)^{s t}$ operation is a delete, it is straightforward to show that all violations are again £xed, by a proof similar to that of Lemmas 3 and 4 . We have thus proved that the invariants hold after any sequence of inserts and deletes.

Corollary 3.1. For the Threshold algorithm, the imbalance ratio $\sigma$ is $\delta^{3}$ after any sequence of inserts and deletes.

Corollary 3.2. For the Fibbing algorithm, the imbalance ratio $\sigma$ is $\phi^{3}$ after any sequence of inserts and deletes.

Theorem 4. For the Threshold (and Fibbing) algorithm, the amortized cost of both inserts and deletes is constant.

Proof. We bound the amortized costs of insert and delete by using the potential method. Let $\bar{L}$ denote the current average load. Consider the potential function $\Phi=$ $c\left(\sum_{i=1}^{n} L\left(N_{i}\right)^{2}\right) / \bar{L}$, where $c$ is a constant to be specifed later. We will show the following: (a) the cost of NBRADJUST is bounded by the drop in potential accompanying it, (b) the cost of REORDER is bounded by the drop in potential accompanying it, and (c) the gain in potential after a tuple insert or delete, before any rebalancing actions, is bounded by a constant. The above three statements together imply that the amortized costs of tuple insert and delete are constant.

NBRADJUST: Recall that a NBRADJUST operation occurs between two nodes whose load differs by at least a factor $\delta$. Let the loads of the two nodes involved be $x$ and $y$. The drop in potential $\Delta \Phi$ from NBRADJUST is $c\left(x^{2}+y^{2}-\right.$ $\left.(x+y)^{2} / 2\right) / \bar{L}=c(x-y)^{2} / 2 \bar{L}$. By Lemma $1(\mathrm{f}), x-y>$ $(\delta-1) y$, and $y$ is at least $\bar{L} / \delta^{3}$. Therefore, $\Delta \Phi>c^{\prime}(x-y)$ for some constant $c^{\prime}$. Since the number of tuples moved is at most $(x-y) / 2$, the drop in potential pays for the data movement by choosing the constant $c$ to be suffciently large $\left(>\delta^{3} /(\delta-1)\right)$.

REORDER: Let a REORDER operation involve a node with load $x$, and a pair of neighbor nodes with loads $y$ and $z$, 
with $y \leq z$. We then have $\delta^{2} y \leq x$ (for the REORDER operation to be triggered), and $\delta z \leq x$ (by NBRBALANCE between the neighbors).

The drop in potential from REORDER is given by:

$$
\begin{aligned}
\Delta \Phi & =c\left(x^{2}+y^{2}+z^{2}-2(x / 2)^{2}-(y+z)^{2}\right) / \bar{L} \\
& =c\left(x^{2} / 2-2 y z\right) / \bar{L} \geq c\left(x^{2} / 2-2 x^{2} / \delta^{3}\right) / \bar{L} \\
& \geq c^{\prime} x\left(1-4 / \delta^{3}\right)
\end{aligned}
$$

Note that $1-4 / \delta^{3}$ is greater than zero for $\delta>\sqrt[3]{4} \simeq 1.587$. The data movement cost of REORDER is $\lfloor x / 2\rfloor+y<x$. Therefore, for a suitable choice of constant $c\left(>2 \delta^{3} /\left(\delta^{3}-\right.\right.$ $4)$ ), the drop in potential pays for data movement.

Tuple Insert: The gain in potential, $\Delta \Phi$, from an insert at node $N_{i}$ and before any rebalancing, is at most $c\left(\left(L\left(N_{i}\right)+\right.\right.$ $\left.1)^{2}-L\left(N_{i}\right)^{2}\right) / \bar{L}$, where $\bar{L}$ refers to the average load before the latest insert. Therefore, $\Delta \Phi \leq c\left(2 L\left(N_{i}\right)+1\right) / \bar{L} \leq$ $c\left(2 \delta^{3}+3\right)$, since $L\left(N_{i}\right) \leq \delta^{3} \bar{L}+\overline{1}$ and $\bar{L} \geq 1$. Therefore, the amortized cost of an insert is constant.

Tuple Delete: When a tuple is deleted, there may be a gain in potential due to a slight reduction in $\bar{L}$. Since $\bar{L}$ drops in value $1 / n$ from a delete, the maximum gain in potential $\Delta \Phi=\frac{c\left(\sum L\left(N_{i}\right)^{2}\right)(1 / n)}{\bar{L}(\bar{L}-1 / n)}$. Using the facts $L\left(N_{i}\right) \leq \delta^{3} \bar{L}+1$, $\bar{L} \geq 1$, and $n \geq 2$, we can see that $\Delta \Phi \leq c\left(5 \delta^{3}+3\right)$. Therefore, the amortized cost of a delete is constant.

We observe that the bounds on these amortized costs are quite large. When $\delta=\phi$, the cost of an insert $\simeq 412$, and the cost of a delete $\simeq 868$. We believe this to be a consequence of weak analysis stemming from our choice of potential function. We show experimentally in Section 5 that these constants are actually very close to 1 . We also present variations of our algorithm next that are amenable to tighter analysis.

\subsection{Discussion}

Improving $\sigma$ further It is possible to improve $\sigma$ to arbitrarily small values larger than 1 , by generalizing the Threshold algorithm, and maintaining balance over larger sets of consecutive nodes, rather than just pairs of neighbors. We do not detail this generalization in this work.

The Doubling Algorithm with Hysteresis It is possible to defne a variant of the Doubling Algorithm which provides a weaker imbalance ratio $(\sigma=32)$ but has provably stronger bounds on the insertion and deletion costs. The idea is to use hysteresis, and require a node to lose at least half its data before it triggers load balancing for tuple deletions. We can show that this variant guarantees insertion cost of 4 and deletion cost of 29 .

A Randomized Variant So far, all our algorithms attempt to fnd the least-loaded node (or the most-loaded node) in order to initiate the REORDER operation. In fact, the theorems we have stated hold even for a slightly weaker condition: If there are multiple nodes that violate the GLOBALBALANCE condition with respect to a particular node $N_{i}$ executing ADJUSTLOAD, it suf£ces for $N_{i}$ to attempt the REORDER operation with any of these nodes.

Such a weakening suggests an interesting randomization which avoids trying to £nd the least-loaded node altogether. Node $N_{i}$ simply samples a set of $\rho$ nodes at random. If one of them violates GLOBALBALANCE, $N_{i}$ performs the REORDER operation using this node; otherwise, $N_{i}$ simply does nothing.

If there are no data deletes, this randomized algorithm guarantees that the maximum load is at most a constant factor times the average load with high probability, so long as the number of nodes sampled $\rho$ is $\Theta(\log n)$. In the presence of tuple deletes, we offer a different guarantee: If a node $N_{i}$ specifes a peak threshold $C$ that $N_{i}$ does not want its load to exceed, the load of $N_{i}$ does not exceed $C$ with high probability, unless the average load in the system $\bar{L}$ is within a constant factor of $C$. It is also possible to provide guarantees on the imbalance ratio in the presence of deletes, but more caveats need to be added to the algorithm. ${ }^{4}$

Concurrency Control and Parallelism Until now, we have assumed that tuple inserts (and deletes) happen in sequence, and that a load-balancing step completes before the next insert. Our algorithms generalize naturally to (a) deal with parallel inserts that may happen before a loadbalancing step completes, and (b) allows multiple loadbalancing steps to execute in parallel.

While we do not discuss either of the above issues in detail here, we make the following observations and claims. First, the load-balancing step can be broken into multiple, smaller, atomic actions that require only simple block-level locking. Second, tuple inserts and deletes may be given higher priority, and allowed to execute even before the full load-balancing step for a previous insert/delete completes. Third, multiple load-balancing steps can execute in parallel and require very simple serialization mechanisms to ensure correctness. Finally, we note that it is possible to formalize a model of concurrency in which we can characterize the imbalance ratio under parallel insertions and deletions.

\section{A P2P Network for Range Queries}

There has been recent interest in developing P2P networks that can support ad-hoc queries over key ranges [3, 4, 25]. A solution is to use range partitioning of data across the peer nodes. If the data and query distributions are uniform, nodes will have equal loads. However, if the data and/or execution is skewed, the network will develop hot-spots with high query traf£c for a few nodes. Load balancing thus becomes a critical requirement in such a system. The $\mathrm{P} 2 \mathrm{P}$ environment imposes three signi£cant challenges for developing a load-balanced, range-partitioned system:

Scale The size of the system may be extremely large, upto tens or hundreds of thousands of nodes. Our load-balancing

\footnotetext{
${ }^{4}$ Karger and Ruhl [17] offer a randomized algorithm that provides such guarantees, but require each node to perform such random sampling and rebalancing, whether or not any inserts or deletes are directed to that node.
} 
algorithm deals well with scale, since its data-movement cost is constant and independent of the number of nodes.

Dynamism The lifetime of nodes is short (a few hours) and arbitrary (whims of the node owner). Our load-balancing algorithms need to effciently handle dynamic arrival and departure of nodes while ensuring good load balance across the existing nodes in the system. We discuss this adaptation in Section 4.1.

Decentralization P2P systems do not have a central site that collects statistics and routes queries/inserts/deletes. We need distributed routing and data structures to enable both routing of queries/inserts/deletes, as well as to £nd additional load information for load balancing. Maintaining such data structures also imposes additional costs on the system, as we discuss in Section 4.2.

\subsection{Handling Dynamism in the Network}

Node Arrival Upon arrival, a new node $N$ £nds the mostloaded node $N_{h}$ in the network. It then splits the range of $N_{h}$ to take over half the load of $N_{h}$, using the NBRADJUST operation. After this split, there may be NBRBALANCE violations between two pairs of neighbors: $\left(N_{h-1}, N_{h}\right)$ and $\left(N, N_{h+1}\right)$. In response, ADJUSTLOAD is executed, $\mathrm{frst}$ at node $N_{h}$ and then at node $N$. It is easy to show (as in Lemma 3) that the resulting sequence of NBRADJUST operations repair all NBRBALANCE violations.

Node Departure While in the network, each node manages data for a particular range. When the node departs, the data it stored becomes unavailable to the rest of the peers. P2P networks reconcile this data loss in two ways: (a) Do nothing and let the "owners" of the data deal with its availability. The owners will frequently poll the data to detect its loss and re-insert the data into the network. (b) Maintain replicas of each range across multiple nodes. A common scheme for replication is to ensure that the partition of node $N_{i}$ is replicated at the preceding $r$ nodes (with $N_{n}$ preceding $N_{1}$ ), for a system-specifed constant $r[9,23]$.

First, consider the simpler data-is-lost case (a). Here, when a node $N_{i}$ departs, the range boundaries between $N_{i-1}$ and $N_{i+1}$ must be modifed. There could be a NBRBALANCE violation between the new neighbors $\left(N_{i-1}, N_{i+1}\right)$ which can be £xed by $N_{i-1}$ executing ADJUSTLOAD. As shown in Lemma 3 , this is suffcient to restore the system invariants.

Now consider the data-is-replicated case (b). Here, when a node $N_{i}$ departs the network, its preceding node $N_{i-1}$ assumes management of $N_{i}$ 's partition. The node $N_{i-1}$ already has $N_{i}$ 's data replicated locally. We can consider the new state as being logically equivalent to a node departure in the data-is-lost case (b), followed by a subsequent insertion of the "lost" tuples by $N_{i-1}$. The loadbalancing algorithm is initiated whenever such insertion makes a node's load cross a threshold.

The Costs of Node Arrival and Departure The datamovement cost of a node arrival and departure is straightforward to analyze. When a new node arrives, it receives half the load of the largest node, thus requiring $\Theta(\bar{L})$ data movements, where $\bar{L}$ is the average load per node after node arrival (since all node loads are within a constant factor of each other). In addition, the average load per node decreases, leading to an increase in potential of $\Theta(\bar{L})$. Thus, the amortized cost of node insertion is still $\Theta(\bar{L})$. Note that this cost is asymptotically optimal, since it is impossible to achieve a constant imbalance ratio without the new node receiving at least $\Theta(\bar{L})$ tuples.

In the data-is-lost case, the data-movement cost of a node departure is 0 , since a node departure only raises the average load, resulting in a drop in potential. All subsequent NBRADJUST operations pay for themselves, as discussed earlier. In the data-is-replicated case, the datamovement cost of a node departure is equal to the cost of "re-insertion" of the "lost" data; since the amortized cost of each insert is constant, and we re-insert only $O(\bar{L})$ tuples, the amortized cost of node departure is $O(\bar{L})$. This is again asymptotically optimal.

Note that in the replicated case, both arrival and departure of nodes requires re-creation of lost replicas, or migration of existing ones. Similarly, tuple inserts and deletes also have to be duplicated at the replica nodes. We presume that such replication is performed in the background. Observe that such replica maintenance inates the costs of all operations only by a constant factor, if the number of replicas $r$ is a constant.

\subsection{Dealing with Decentralization}

So far, we have assumed the existence of a central site that (a) maintains the global partitioning information to direct queries appropriately, and (b) maintains global load information for the load-balancing algorithm to exploit. Our next step is to devise decentralized data structures to perform both the above functions. We frst describe a known data structure for ef£cient range queries, before discussing how to maintain load information.

Cost Model In the centralized setting of Section 2, we considered only data-movement cost, and ignored the cost of maintaining partition and load information. In the P2P setting, the lack of a central site means that we can no longer ignore this latter cost. Therefore, each operation (query, tuple insert, tuple delete, node insert, node delete) is now associated with two different costs: (a) the data-movement cost, which is measured just as earlier, and (b) communication cost, de£ned to be the number of messages that need to be exchanged between nodes (to maintain and probe the data structure) in order to perform the operation (and its corresponding load-balancing actions). Note that each message is between a pair of nodes, i.e., communication is point-to-point and broadcast is not free. (We will ignore the cost of returning query answers to the querying node.)

A Data Structure for Range Queries Our £rst task is the following: Any node receiving a query/insert/delete should be able to ef£ciently forward the operation to the appropriate node(s). One solution is to replicate the partition 
information across all nodes. This enables any node to directly forward a query to the relevant nodes. However, every node join/leave, or partition change, needs to be broadcast to all nodes, which is very inef£cient. On the other extreme, nodes could be organized in a linked list, ordered by the partitions they manage. Updating the data structure on partition changes or node arrival/departure is ef£cient, but queries may have to traverse the entire linked list to reach the relevant node.

A compromise between these two costs may be achieved using a data structure known as the skip graph [6, 16]. Skip graphs are essentially circular linked lists, but each node also maintains roughly $\log n$ skip pointers, to enable faster list traversal. Skip pointers are randomized, and routing between any two nodes requires only $O(\log n)$ messages with high probability. Consequently, a query can be forwarded from any node to the frst node in the query's range, say $N_{1}$, using $O(\log n)$ messages. If the query range is large and spans $q$ nodes, the query is simply forwarded along on the linked list to the $q$ successors of $N_{1}$, using a total of $O(\log n+q)$ messages. When a node arrives or departs, only $O(\log n)$ messages are required to update the data structure. Partition changes due to NBRADJUST do not require any messages at all. Thus, queries, node joins/leaves and load balancing actions are all ef£cient.

Maintaining Load Information Our algorithm requires that each node be able to £nd (a) the load of its neighbors, and (b) the most and least-loaded node in the system. Dealing with problem (a) is easy: a node already has links to its two neighbors in the skip graph, thus requiring just one message each to fnd their loads.

To deal with problem (b), we simply build a second, separate skip graph on the node loads. In other words, nodes are arranged in a sequence sorted by their current load (with ties broken arbitrarily), and a skip graph is constructed on this sequence. As node loads change, the sequence may have to be updated, but it will turn out that such updates are not expensive. As discussed earlier, this data structure enables discovery of the most and least-loaded node with just $O(\log n)$ messages, while also enabling ef£cient updates to the data structure.

As mentioned in Section 3.4, it is not necessary for a node to always $£$ nd the most or least-loaded node, so long as it locates any node that violates GLOBALBALANCE. This property allows us to terminate searches on the skip graph even before locating the most or least-loaded node. The early termination mitigates "hot spots" created when multiple nodes simultaneously seek the most-loaded node.

The P2P Structure and its Costs: A Summary We summarize all the operations supported by the P2P system and their costs in Table 4.2. The bounds on the message costs of operations follow directly from our discussion of skip graphs above. We note that the data-movement costs are amortized, while the message costs hold with high probability. We observe that the above costs are asymptotically identical to the costs in DHTs, except for range queries where our structure is more ef£cient.

\begin{tabular}{|l|c|c|}
\hline Operation & Messages (w.h.p) & Data Movement \\
\hline Tuple Insert & $O(\log n)$ & $O(1)$ \\
Tuple Delete & $O(\log n)$ & $O(1)$ \\
Node Arrival & $O(\log n)$ & $O(\bar{L})$ \\
Node Departure & $O(\log n)$ & 0 or $O(\bar{L})$ \\
Lookup Query & $O(\log n)$ & 0 \\
\hline Range Query & $O(\log n+f n)$ & 0 \\
\hline
\end{tabular}

Table 1: Cost of operations supported by the P2P network. The parameter $f$ denotes the selectivity of the range query. The data-movement cost of Node Departure depends on the model used for data loss.

\section{Experimental Evaluation}

In this section, we present results from our simulation of the Threshold algorithm on networks ranging in size from $n=2^{4}$ to $2^{14}$. We compare the performance of our algorithm against periodic reorganization. We also evaluate our adaptations of the algorithm on a P2P network. Our simulations show the following results:

A The Threshold Algorithm achieves the desired imbalance ratio for a range of $\sigma$ values on various workloads.

B The amortized cost of load balancing is very small, decreases with increasing $\sigma$, and is much lower than the cost of periodic reorganization.

$\mathrm{C}$ The P2P variant achieves the desired imbalance ratio at a small cost, and scales gracefully with increasing dynamism in the network.

D The Randomized variant provides good imbalance ratios even with a small number of samples.

\subsection{Simulation Model}

In the parallel database setting, the simulation is designed to study load balancing as the relation evolves over time. The system is studied under three phases: (a) Growing, (b) Steady, and (c) Shrinking. At the start, all $n$ nodes in the system are empty ("cold start"). In the Growing phase, data is loaded, one tuple at a time, using a sequence of $D=10^{6}$ insert operations. In the Steady phase, inserts and deletes alternate for a total of $D$ operations. In the following Shrinking phase, data is removed from the system, one tuple at a time, using a sequence of $D$ delete operations.

The workload, i.e., the sequence of insertions and deletions, is set to be one of the following:

A ZIPFIAN models a static data distribution. Each tuple inserted in the Growing and Steady phases has an attribute $A$ drawn from a Zip£an distribution (with parameter 1.0$)$ with values in the range $[1,10000]$. (Since our range partitioning operates on a relational key, we create a unique attribute $B$ for each tuple, and use the sequence $\langle A, B\rangle$ as the ordering key for range partitioning.) Tuple deletion during the Steady and Shrinking phases removes one of the existing tuples uniformly at random.

B HotSpot models a skewed workload in which all inserts and deletes are directed to a single pre-selected ("hot") node.

C SHEARSTRESS models a dynamic workload in which 

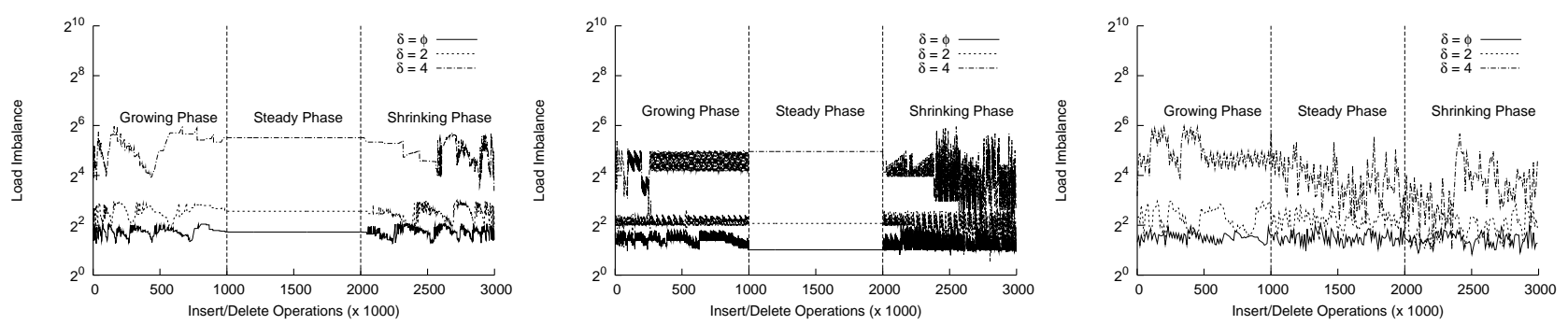

Figure 3: Imbalance ratio for (a) ZipfiAn, (b) HotSpot, and (c) SHEARSTRESS when $n=256$.
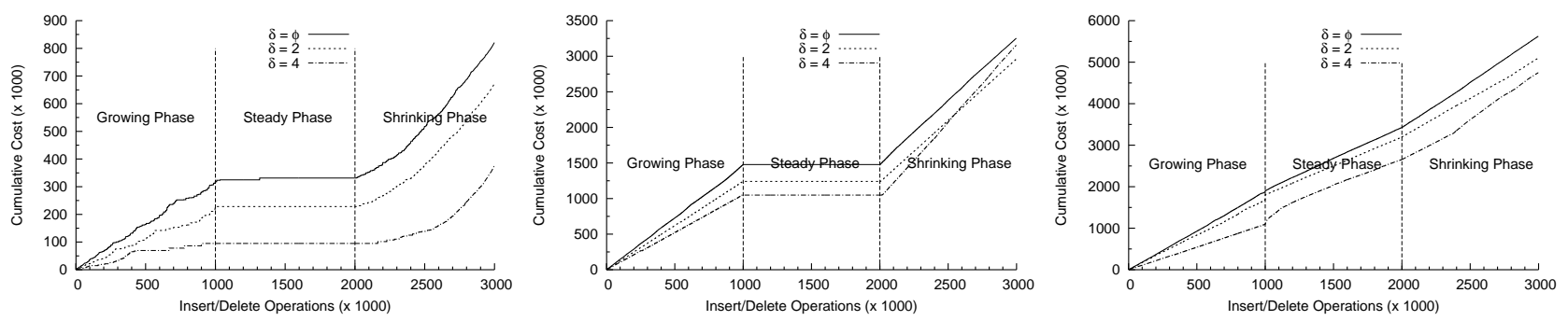

Figure 4: Data movement costs on the (a) ZiPfiAn, (b) HOTSPOT, and (c) SHEARSTRESS workload when $n=256$.

an "adversary" inspects the load of nodes after each insert or delete of a tuple. The adversary then constructs the following insert (or delete) such that it is routed to the current most-loaded (resp. least-loaded) node.

We study the effects of network dynamism on loadbalancing for a P2P network under a similar evolution model. The network starts with an initial $n=16$ nodes, into which $D$ tuples are inserted one by one. In the Growing phase, nodes arrive one by one and join the network, until $n=1024$. In the following Shrinking phase, nodes depart at random until the network shrinks to $n=16$. We use data replication to ensure that tuples are not lost on node departures. No tuples are inserted or deleted during the Growing and Shrinking phases: our goal is to isolate the costs of node arrival/departure on load balancing.

\subsection{Imbalance Ratio}

We start with an evaluation of imbalance ratios ensured by the Threshold Algorithm for various workloads. For the experiments, we measure the imbalance ratio at any instant as the ratio of the largest and smallest loads at that instant (with all loads being at least 1). Figure 3 shows the imbalance ratio (Y-axis) against the number of insert and delete operations (X-axis) during a run on (a) ZIPFIAN, (b) HotSPOT, and (c) SHEARSTRESS workloads with 256 nodes. The curves are drawn for $\delta=\phi$ (Fibbing Algorithm), $\delta=2$ (Doubling Algorithm) and $\delta=4$.

We observe that Threshold Algorithm ensures that imbalance ratio is always less than $\delta^{3}$ for all $\delta$. Each spike in the curve corresponds to an ADJUSTLOAD step. As $\delta$ increases, the jitter introduced by the spikes gets larger and larger; this is because the algorithm allows the imbalance ratio to worsen by a constant factor, roughly $\delta$, before load balancing occurs. The curves are smooth in the Steady phase for ZIPFIAN and HOTSPOT. For the former, the range partitioning "adapts" to the data distribution, ensuring that inserts and deletes are randomly sprinkled across nodes; for the latter successive inserts and deletes occurring at the same node cancel out. However, the adversary in SHEARSTRESS picks its inserts and deletes carefully to cause imbalance, leading to continuous variation in $\sigma$.

\subsection{Data Movement Cost}

We next study the data movement cost incurred by the Threshold Algorithm for ensuring balance in the runs discussed above. Figure 4 plots the cumulative number of tuples moved by the algorithm (Y-axis) against the number of insert and delete operations ( $\mathrm{X}$-axis) during a run.

We observe that costs for different $\delta$ are roughly the same (within $20 \%$ of each other) for the НотSPOT and SHEARSTRESS workloads. Intuitively, this is because keeping the system tightly balanced causes a larger number of rebalancing operations, but each operation has lower cost due to the tight balance. We also observe that there is no data movement in the Steady phase for ZIPFIAN, indicating that the system has "adapted" to the data distribution. For the other two phases, the curves are linear confrming that the amortized cost per operation is constant, and independent of the amount of data in the system. The constants involved are also very small, with the cost per insert/delete, even in the worst phase, being roughly $0.3,1.5$ and 2 for the three workloads.

To put the above performance in perspective, we compared the data movement costs of the Fibbing Algorithm against those incurred by a periodic reorganization strategy that ensures the same imbalance ratio $\sigma=4.2$ bound as follows: the central site continuously observes $\sigma$, and whenever $\sigma>4.2$, a reorganization is triggered to create a perfectly balanced set of nodes. The reorganization identifes a balanced placement of tuples across nodes, and then 

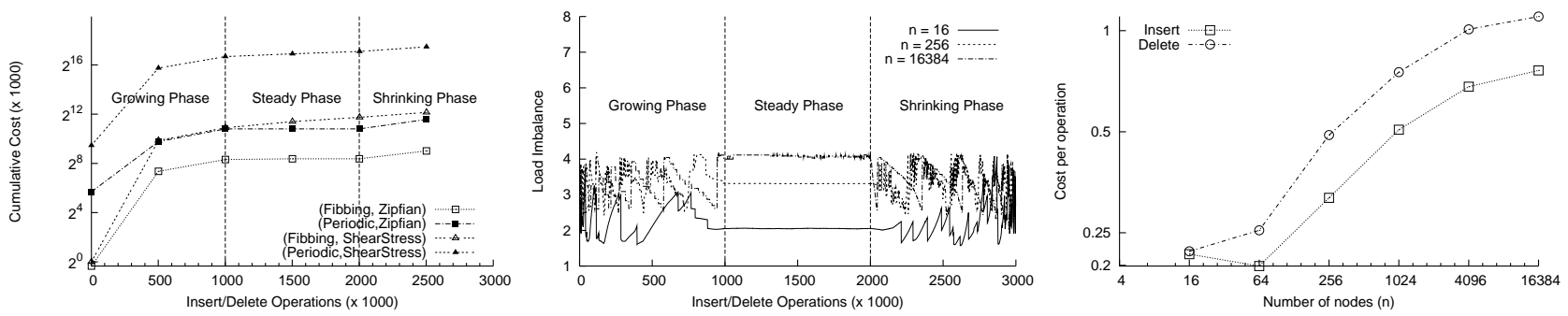

Figure 5: (a) Data movement costs for Fibbing Algorithm compared to periodic reorganization (b) Effect of $n$ on imbalance ratios (c) Effect of $n$ on data movement cost.
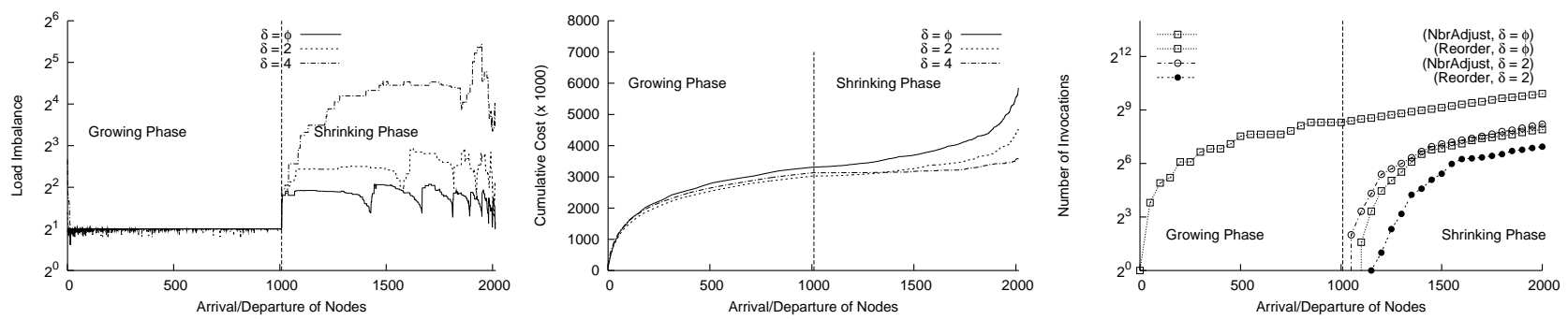

Figure 6: Performance in a P2P system: (a) imbalance ratios, (b) data movement costs, and (c) number of NBRADJUST and REORDER invocations for the Threshold Algorithm on the ZIPFIAN workload.

moves each tuple at most once by sending it directly to its Enal destination node. (Thus, it is more effcient than using only NBRADJUST operations.) Figure 5(a) plots the cumulative data movement costs on a logarithmic scale (Yaxis) against the number of operations (X-axis) in a run on 256 nodes. We observe that the periodic reorganization performs nearly 10 times worse for ZIPFIAN and upto 50 times worse for others. The reasons are two-fold: (a) its non-online nature allows the skew to grow requiring an expensive clean-up, and (b) its perfect balancing causes more data movement than essential to obtain the desired bounds.

\subsection{The Effects of Scaling}

We next study the effects of scaling in the number of nodes $n$ on the performance of the Threshold Algorithm. Figures 5(b) and 5(c) plot load imbalance and data movement cost for the Fibbing Algorithm against a run on the ZIPFIAN workload. The network size $n$ is varied from 16 to 16384. We observe in Figure 5(b) that the Fibbing Algorithm continues to ensure the $\sigma=\phi^{3}$ bound. However, as the same number of tuples are shared across more nodes, the load variance across nodes increases, leading to an increase in the imbalance ratio.

Figure 5(c) plots the data movement cost per operation (Y-axis) against the size of the network $n$ (X-axis). Both axes are plotted on a logarithmic scale. The bottom curve plots the data movement cost per insert observed during the Growing Phase; the top curve plots the costs per delete observed during the Shrinking Phase. We observe that costs of both insert and delete operations increases with increasing $n$. As $n$ increases, the load per node is smaller, making it easier to make the system unbalanced with a smaller number of inserts and deletes. Thus more load balancing steps are needed, leading to a higher cost. We also observe that the cost per operation is quite small as the curves taper off towards a value close to 1 .

We had shown in Section 3.3 that the cost per insert or delete is a constant. The fgures here show a dependence of cost per operation on $n$. How can this apparent contradiction be explained? The experiments presented here evaluate the cost for a $£$ xed workload for various $n$ values. On the other hand, the analysis established bounds on the worst-case costs against all workloads.

\subsection{Performance in a P2P Setting}

Figure 6 shows the performance of the Threshold Algorithm adapted to a P2P system. Figures 6(a) and 6(b) plot the imbalance ratio and data-movement cost against the number of node arrivals and departures. We observe in Figure 6(a) that the system remains well-balanced through the Growing phase, because the arriving node always splits the most-loaded node. The imbalance ratio is roughly two, since the most-loaded node splits its load in half on a node arrival. On the other hand, nodes depart at random during the Shrinking phase, which leads to changes in the imbalance ratio. However, the guarantees of $\delta^{3}$ are ensured.

From Figure 6(b), we see that the incremental datamovement cost per arrival/departure (i.e., the slope of the curve) decreases with node arrivals in the Growing phase, and increases with node departures in the Shrinking phase. This is not surprising, since the cost is proportional to the average load in the system which, in turn, is inversely proportional to the number of nodes.

NbrAdjust vs. Reorder In a P2P system, the NBRADJUst operation may turn out to be more ef£cient than REORDER, 

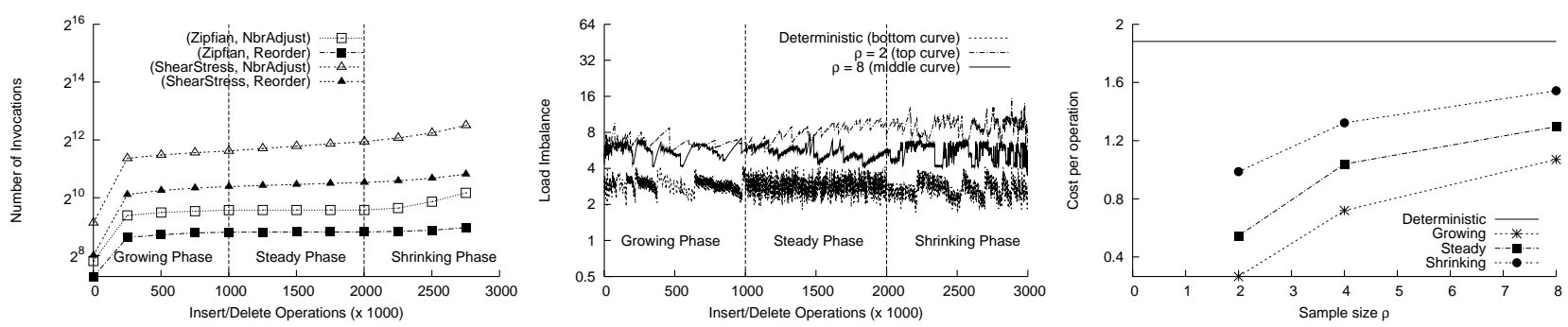

Figure 7: The Fibbing algorithm with $n=256$. (a) The number of invocations of NBRADJUST and REORDER (b) The effect of randomization on imbalance ratio (c) The effect of randomization on data movement costs, for the SHEARSTRESS workload.

for two reasons: (a) REORDER requires the reordered node to drop its old links and set up new ones. (b) A NBRADJUST may not require immediate transfer of data to balance load, when data is replicated at neighbors; only replicas need to be updated, which can be done more lazily.

In the light of the above observations, we observed the number of invocations of the two operations to see which is used more often. Figure 6(c) shows the number of invocations of NBRADJUST and REORDER for different values of $\delta$, as nodes are inserted and deleted. Not surprisingly, the number of invocations of both operations decreases as $\delta$ increases. We see that the number of NBRADJUST invocations is at least 4 times that of REORDER, which is reassuring given that REORDERs are more expensive.

Figure 7(a) shows the number of invocations of the two operations by the Fibbing Algorithm, on a £xed set of 256 nodes, as tuples are inserted and deleted from the three workloads. We observe that there are twice as many invocations of NBRADJUST, as compared to REORDER, which is again useful in the $\mathrm{P} 2 \mathrm{P}$ context.

\subsection{The Effects of Randomization}

As discussed earlier, the REORDER operation requires global statistics and involves the least/most-loaded node in the load-balancing step. In Section 3.4, we de£ned a randomized variant where REORDER would sample $\rho$ nodes at random and pick the least/most-loaded node from the sample. Figures 7(b) and 7(c) plot the effects of such randomization on the Fibbing Algorithm for runs of the SHEARSTRESS workload as the sample size $\rho$ is varied. We observe in Figure 7(b) that the imbalance ratio degrades beyond the original value of $\phi^{3}$. However, even the use of $\rho=2$ samples provides good load balance; increasing $\rho$ improves $\sigma$ further. Correspondingly, the use of sampling reduces data movement compared to the deterministic case, for all three phases, as shown in Figure 7(c).

\section{Related Work}

Parallel Databases partition relations across multiple disks, using either range or hash partitioning [8, 10, 28]. Research in physical design of parallel databases can be classi£ed into four categories: (a) Workload-driven tuning of storage for static relations, e.g. [15, 21, 26]; (b)
Disk-based data structures for fast bulk insert/delete of tuples, e.g. [12, 18]; (c) Effcient data migration for loadbalancing while allowing concurrent relation updates and queries, e.g. [29]; and (d) Balancing query load across disks by moving a partition from one disk to another, e.g. [24].

Work in category (a) is focused on performing workload-driven tuning of physical design, but usually does not consider a dynamic evolution of the design with relation updates. Work in category (b) is complementary to ours, in that they show how to ef£ciently update local disk structures when tuples move from one partition to another, while our focus is in understanding what tuples to move. Research in category (c) is also complementary to our work, as it helps deal with issues of concurrency control when performing online repartitioning. Finally, work in category (d) attempts to modify the allocation of partitions to disks, rather than change the partitions themselves. We believe such solutions could be used in combination with ours to achieve balance for dynamic query loads, but are not suffcient in themselves to guarantee storage balance for range-partitioned data.

Litwin et al. [20, 19] consider the design of scalable distributed data structures (SDDS) which share many features with the design philosophy of $\mathrm{P} 2 \mathrm{P}$ systems, including the absence of centralization, and the ability to gracefully add or remove servers. Most work on SDDS has focused on hash partitioning, either at the tuple level [20] or at a block level [19]. Our work is complementary in that it can be utilized to enable true range partitioning for SDDS.

Range Queries in P2P Networks Recently, P2P networks supporting range queries have been proposed, that offer either storage balance or effcient queries, but not both. Ratnasamy et. al. [25] assure storage balance but at the price of data-dependent query cost and data fragmentation. Gupta et. al. [3] provide approximate answers, and do not offer any guarantees for an arbitrary range query. Others $[4,6,16]$ offer exact and ef£cient queries, but do not offer load balance across nodes.

Aberer et al. [1, 2] develop a P2P network called P-Grid that can support effcient range queries. All nodes in this system have a £xed capacity, and content is heuristically replicated to $£ l l$ all the nodes' capacity. However, there is no formal characterization of either the imbalance ratio guaranteed, or the data-movement cost incurred. 
In a concurrent work, Karger and Ruhl [17] provide an alternative solution to the storage balance problem. The scheme is a randomized algorithm that offers a highprobability bound on the imbalance ratio, and is analyzed under a dynamic, but non-adversarial, setting. However, the best achievable bound on imbalance ratio using this algorithm appears to be more than 128 , which is much higher than the load imbalance bounds we guarantee. Bharambe et al. [7] also use a load-balancing scheme similar to [17] as a heuristic to balance range partitions.

Routing in P2P Networks Most DHT interconnection networks [23, 27] require randomly chosen (uniformly spaced) partition boundaries to guarantee ef£cient routing. A load-balanced, range-partitioned network will not have such equi-spaced boundaries, rendering such networks unusable. Aberer [1] presents an elegant variant of Pastry which does guarantee $O(\log n)$ routing even with arbitrary partition boundaries. However, node in-degrees could become skewed, resulting in a skewed message traf£c distribution. Moreover, a change of partition boundaries between neighbors necessitates a change in the network link structure. Our P2P network utilizes skip graphs and overcomes the above limitations. Bharambe et al. [7] suggest an alternative scheme involving the construction of smallworld networks. However, their heuristic requires nodes to perform extensive sampling of the other nodes in the system, and provides no guarantees on routing performance. Our solution of using skip graphs is simpler and provides stronger guarantees on performance.

\section{Conclusions and Future Work}

Horizontal range-partitioning is commonly employed in shared-nothing parallel databases. Load balancing is necessary in such scenarios to eliminate skew. We presented asymptotically optimal online load-balancing algorithms that guarantee a constant imbalance ratio. The datamovement cost per tuple insert or delete is constant, and was shown to be close to 1 in experiments. We showed how to adapt our algorithms to dynamic P2P environments, and architected a new P2P system that can support ef£cient range queries.

Although our algorithms were presented in the context of balancing storage load, they generalize to balancing execution load too; all that is required is an ability to partition load evenly across two machines. Understanding the costs of load balancing for execution load is a subject of future work. We are currently exploring extensions of our algorithm to deal with node and network heterogeneity, as well as the partitioning of multi-dimensional data [14].

\section{References}

[1] K. Aberer. Scalable data access in $\mathrm{p} 2 \mathrm{p}$ systems using unbalanced search trees. In Proc. WDAS, 2002.

[2] K. Aberer, A. Datta, and M. Hauswirth. The quest for balancing peer load in structured peer-to-peer systems. Technical Report IC/2003/32, EPFL, Switzerland, 2003.
[3] A.Gupta, D.Agrawal, and A. Abbadi. Approximate range selection queries in peer-to-peer systems. In Proc. CIDR, 2003.

[4] A. Andrzejak and Z. Xu. Scalable, ef£cient range queries for grid information services. In Proc. P2P, 2002.

[5] A.Silberschatz, H.F.Korth, and S.Sudarshan. "Database System Concepts", chapter 17. McGraw-Hill, 1997.

[6] J. Aspnes and G. Shah. Skip graphs. In Proc. SODA, 2003.

[7] A. Bharambe, M. Agrawal, and S. Seshan. Mercury: Supporting scalable multi-attribute range queries. In Proc. SIGCOMM, 2004.

[8] G. Copeland, W. Alexander, E. Boughter, and T. Keller. Data placement in Bubba. In Proc. SIGMOD, 1988.

[9] F. Dabek, M. F. Kaashoek, D. Karger, R. Morris, and I. Stoica. Wide-area cooperative storage with CFS. In Proc. SOSP, 2001.

[10] D. DeWitt, R. H. Gerber, G. Graefe, M. L. Heytens, K. B. Kumar, and M. Muralikrishna. Gamma -a high performance datadow database. In Proc. VLDB, 1986.

[11] D. DeWitt and J. Gray. Parallel database systems: The future of high performance database processing. Communications of the ACM, 36(6), 1992

[12] H. Feelia, M. Kitsuregawa, and B. C. Ooi. A fast convergence technique for online heat-balancing of btree indexed database over shared-nothing parallel systems. In Proc. DEXA, 2000.

[13] P. Ganesan, M. Bawa, and H. Garcia-Molina. Online balancing of range-partitioned data with applications to $\mathrm{p} 2 \mathrm{p}$ systems. Technical Report http://dbpubs.stanford.edu/pubs/2004-18, Stanford U., 2004.

[14] P. Ganesan, B. Yang, and H. Garcia-Molina. One torus to rule them all: Multi-dimensional queries in p2p systems. In WebDB, 2004.

[15] S. Ghandeharizadeh and D. J. DeWitt. A performance analysis of alternative multi-attribute declustering strategies. In Proc. SIGMOD, 1992.

[16] N. J. A. Harvey, M. Jones, S. Saroiu, M. Theimer, and A. Wolman. Skipnet: A scalable overlay network with practical locality properties. In Proc. USITS, 2003.

[17] D. R. Karger and M. Ruhl. Simple ef£cient load-balancing algorithms for peer-to-peer systems. In Proc. IPTPS, 2004.

[18] M. L. Lee, M. Kitsuregawa, B. C. Ooi, K.-L. Tan, and A. Mondal. Towards self-tuning data placement in parallel database systems. In Proc. SIGMOD, 2000.

[19] W. Litwin, M.-A. Neimat, and D. A. Schneider. RP* : A family of order preserving scalable distributed data structures. In Proc. VLDB, 1994.

[20] W. Litwin, M.-A. Neimat, and D. A. Schneider. LH* - A scalable, distributed data structure. ACM Transactions on Database Systems, 21(4):480-525, 1996.

[21] J. Rao, C. Zhang, N. Megiddo, and G. Lohman. Automating physical database design in a parallel database. In Proc. SIGMOD, 2002.

[22] S. Ratnasamy, P. Francis, M. Handley, and R. M. Karp. A scalable Content-Addressable Network. In Proc. SIGCOMM, 2001.

[23] A. I. T. Rowstron and P. Druschel. Pastry: Scalable, distributed object location, and routing for large-scale peer-to-peer systems. In Proc. Middleware, 2001.

[24] P. Scheuermann, G. Weikum, and P. Zabback. Adaptive load balancing in disk arrays. In Proc. FODO, 1993.

[25] S.Ratnasamy, J.M.Hellerstein, and S.Shenker. Range queries over DHTs. Technical Report IRB-TR-03-009, Intel, 2003.

[26] T. Stohr, H. Martens, and E. Rahm. Multi-dimensional database allocation for parallel data warehouses. In Proc. VLDB, 2000.

[27] I. Stoica, R. Morris, D. Karger, M. F. Kaashoek, and H. Balakrishnan. Chord: A scalable peer-to-peer lookup service for internet applications. In Proc. SIGCOMM, 2001

[28] Tandem Database Group. Nonstop sql, a distributed highperformance, high-reliability implementation of sql. In Proc. HPTS, 1987.

[29] D. C. Zilio. Physical Database Design Decision Algorithms and Concurrent Reorganization for Parallel Database Systems. $\mathrm{PhD}$ thesis, University of Toronto, 1988. 